\title{
Medically Managed latrogenic Aortic Dissection: A Safer Option for a Dangerous Condition?
}

\author{
Evan D Levine ${ }^{1 *}$, Arber Kodra ${ }^{1}$, Rachel M Bond ${ }^{1}$, Fadi Khoury ${ }^{2}$, Dan Inder S Sraow ${ }^{2}$ and Georges $Y$ \\ Nseir $^{2}$ \\ ${ }^{1}$ Department of Cardiology, Lenox Hill Hospital, New York, USA \\ ${ }^{2}$ Department of Cardiology, Chandler Regional Medical Center, Chandler, Arizona, USA

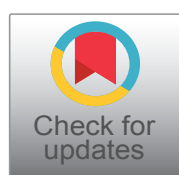

*Corresponding author: Dr. Evan D Levine, Department of Cardiology, Lenox Hill Hospital, 100 E 77th Street, 6 Black Hall, New York, New York 10075, USA, Tel: +845-548-6247, Fax: +212-434-2246, E-mail: Evan.d.levine@gmail.com

\section{Introduction}

latrogenic aortic dissection is a rare complication of both diagnostic and interventional coronary angiography. In the few case studies that have been reported in current medical literature, surgical management was generally opted for; however, with poor overall outcomes. Given these unfavorable outcomes, a different treatment strategy should be considered. We report a case of iatrogenic aortic dissection successfully treated with intensive medical therapy and utilization of a multi-modality serial imaging approach.

\section{Case Report}

A 76-year-old male with known coronary artery disease with a prior history of coronary artery bypass (CABG) surgery, percutaneous coronary intervention, known chronic total occlusion (CTO) of his right coronary artery (RCA) (Figure 1), hypertension and hyperlipidemia, was brought in for elective cardiac catheterization due to exertional anginal symptoms despite optimal medical therapy with three antianginal medications.

Prior to the catheterization, an outpatient nuclear stress test was performed which showed significant RCA ischemia demonstrated by a large area of a severe reversible defect in the inferior wall on SPECT imaging. Additionally, an echocardiogram was performed which was significant for ejection fraction of $45 \%$, no segmental wall motion abnormalities, mild aortic regurgitation and mild pulmonary regurgitation.

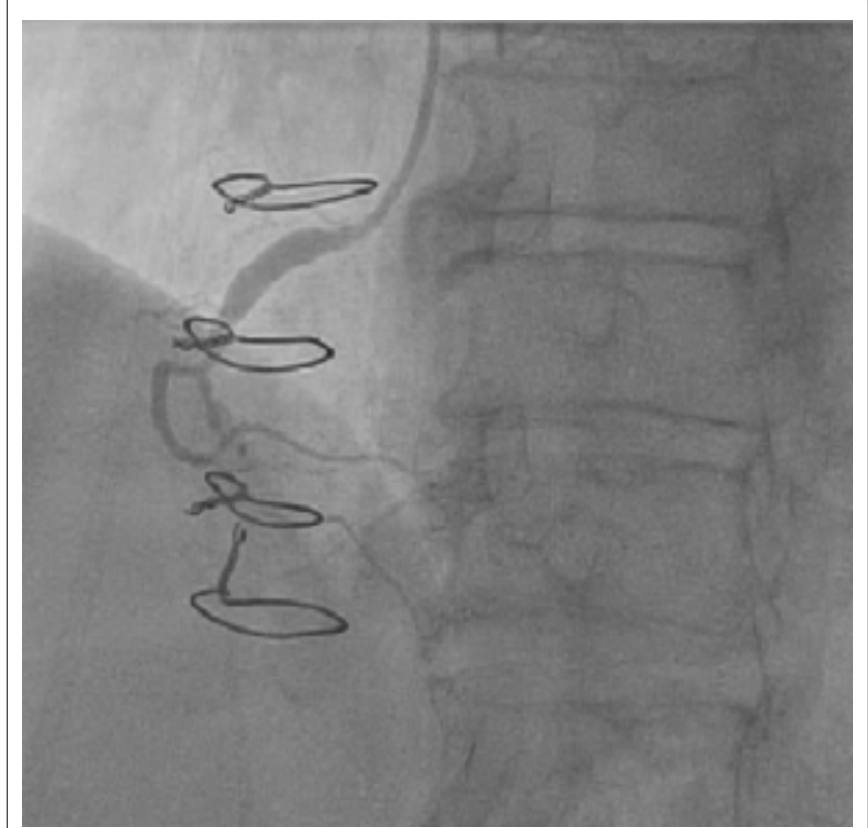

Figure 1: Selective coronary angiography performed onemonth prior demonstrates moderate to severe ostial disease of the RCA with post stenotic dilatation. A chronic total occlusion in the mid segment of the right coronary artery is present.

The planned procedure was to attempt to recanalize the known CTO of the RCA. The RCA was engaged with an 8 French (AL1) guiding catheter with side holes, and a GuideLiner ${ }^{\circledR}$ catheter was advanced over the wire to stabilize the guide into the ostium of the RCA. Selective angiography was performed and showed evidence of an aortic dissection flap at the level of the root adjacent to

Citation: Levine ED, Kodra A, Bond RM, Khoury F, Sraow DIS, et al. (2018) Medically Managed latrogenic Aortic Dissection: A Safer Option for a Dangerous Condition?. Int J Clin Cardiol 5:118. doi. org/10.23937/2378-2951/1410118

Accepted: June 02, 2018; Published: June 04, 2018

Copyright: (C) 2018 Levine ED, et al. This is an open-access article distributed under the terms of the Creative Commons Attribution License, which permits unrestricted use, distribution, and reproduction in any medium, provided the original author and source are credited. 


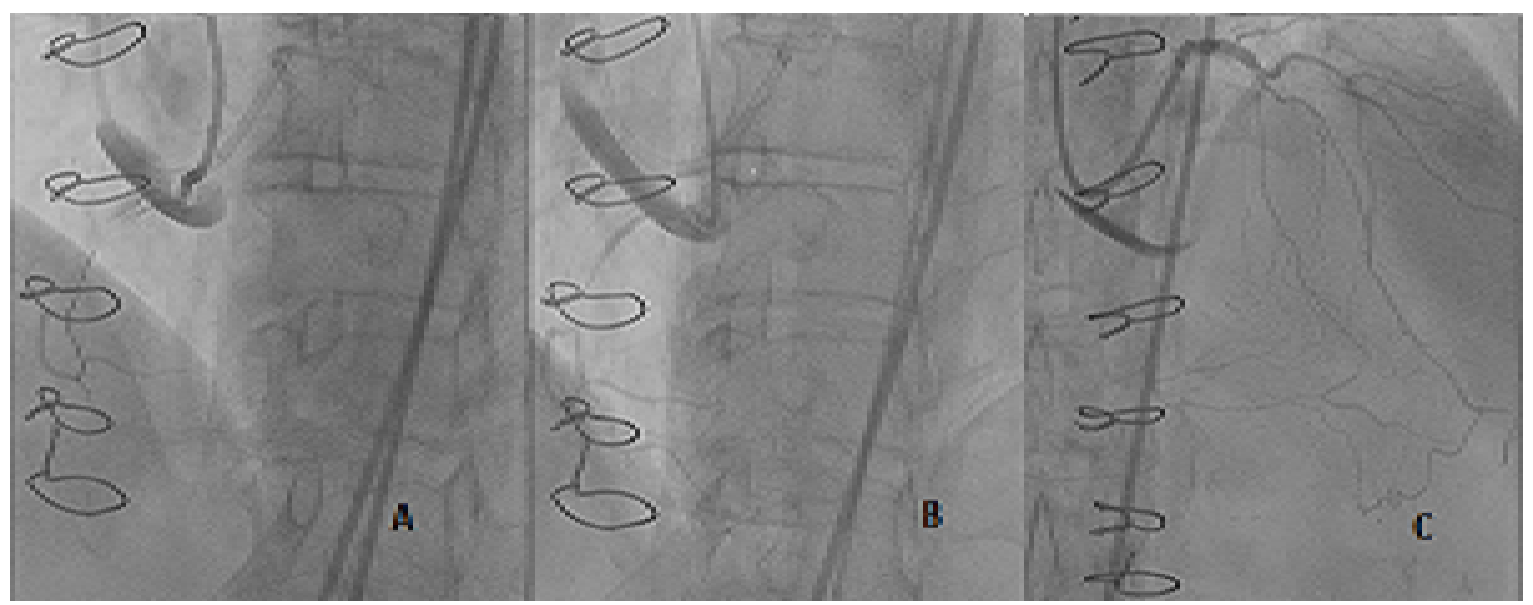

Figure 2: $A, B) R C A$ angiographic appearance: Contrast in the false lumen of the aortic root dissection originating from RCA ostium; C) Angiographic appearance of Left coronary system showing presence of patent collaterals.

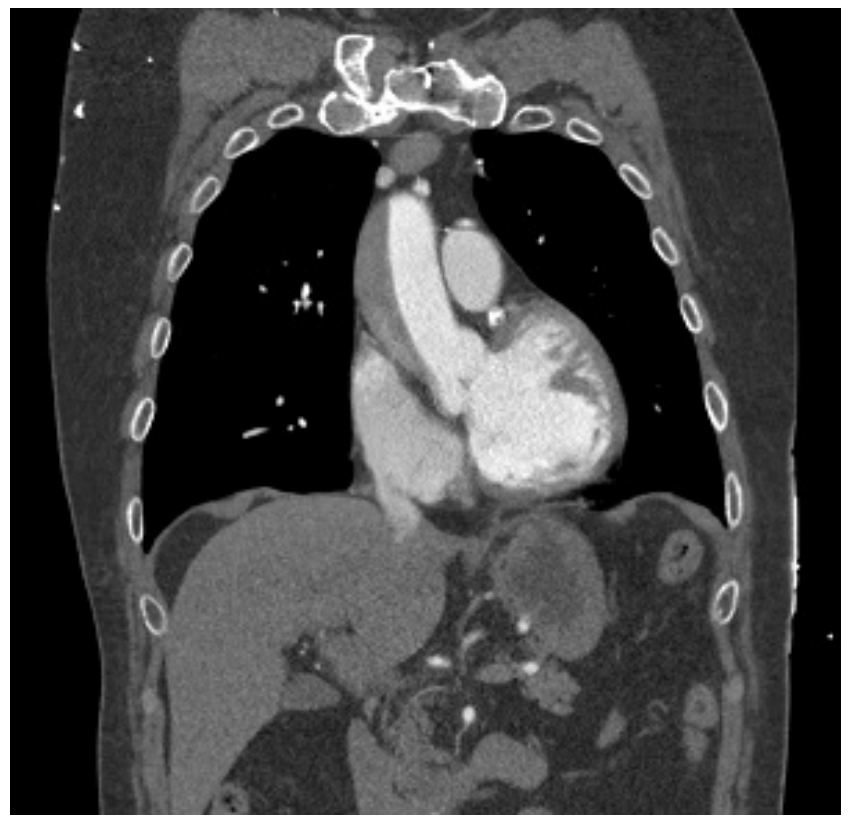

Figure 3: Coronal reformatted CT angiography images performed immediately after the catheterization demonstrating a Stanford type A aortic dissection with dilatation of the ascending aorta measuring $4.6 \mathrm{~cm}$. Extension is seen from the root and ends proximal to the innominate artery. The greatest thickness of the dissection measures $1.9 \mathrm{~cm}$.

the RCA ostium (Figure 2). At this point the patient was stable and asymptomatic. The decision was made to forgo stenting of the RCA ostium and to avoid injecting further contrast due to concern of further propagation of the dissection. The cardiac catheterization procedure was abandoned, and all catheters were removed. Emergent transthoracic echocardiogram was performed and demonstrated no evidence of aortic annular involvement or of pericardial effusion. The patient remained asymptomatic and hemodynamically stable.

CT Angiography was performed immediately after the procedure to assess the extent of the dissection and to confirm a diagnosis of a Stanford Type A aortic dissection (Figure 3 ). The patient was evaluated by cardiothoracic surgery and deemed extreme risk for surgery. The patient was then admitted to the Intensive Care Unit

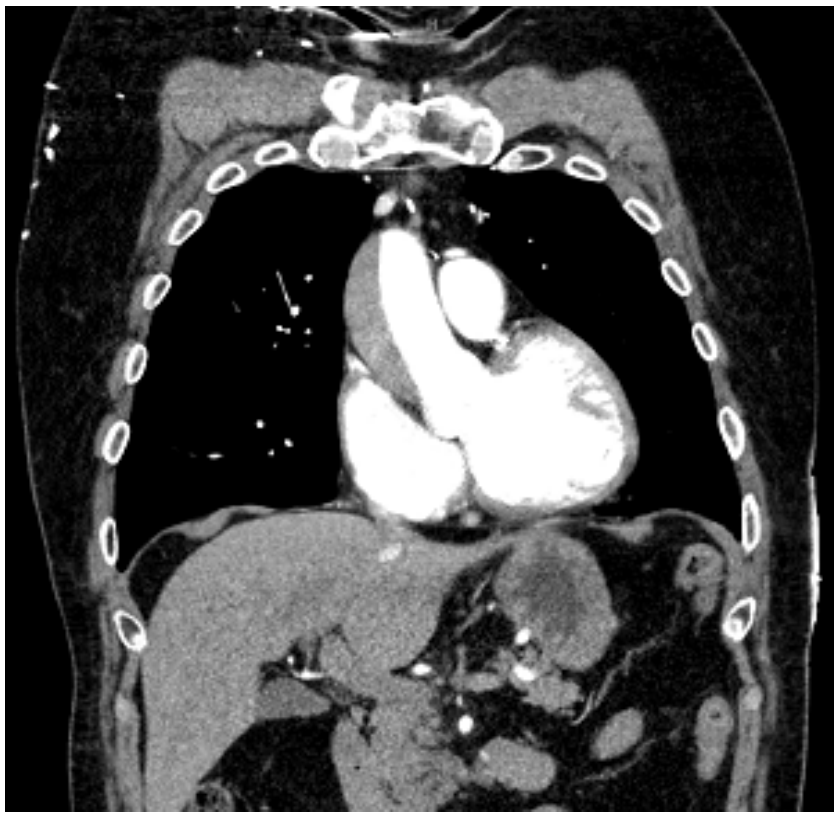

Figure 4: CT angiogram performed 6 hours after demonstrating that the extent of the dissection has worsened in comparison to Figure 2 and extends up to the level of the aortic arch. There is no definite involvement of the brachiocephalic trunk, left common carotid or subclavian artery origins. The greatest thickness of the dissection measures approximately $2.3 \mathrm{~cm}$. The descending thoracic aorta diameter is unchanged.

and the case discussed by the integrated heart team. A decision was made to monitor and treat the patient clinically with the use of intensive medical therapy, as opposed to surgical intervention.

As the patient was already maximally beta-blocked with a heart rate $(\mathrm{HR})<60 \mathrm{bpm}$, the patient was started on a nitroglycerine infusion. As his HR reflexively mildly increased, an esmolol infusion was initiated to maintain a $\mathrm{HR}<60 \mathrm{bpm}$ and $\mathrm{SBP}<120 \mathrm{mmHg}$ with the intent to lower shear forces. A repeat $\mathrm{CT}$ angiogram completed six hours later showed anterograde progression of the dissection flap (Figure 4). A final repeat CT angiogram 12 hours later showed near complete resolution of dissection without further intervention (Figure 5). Patient was 


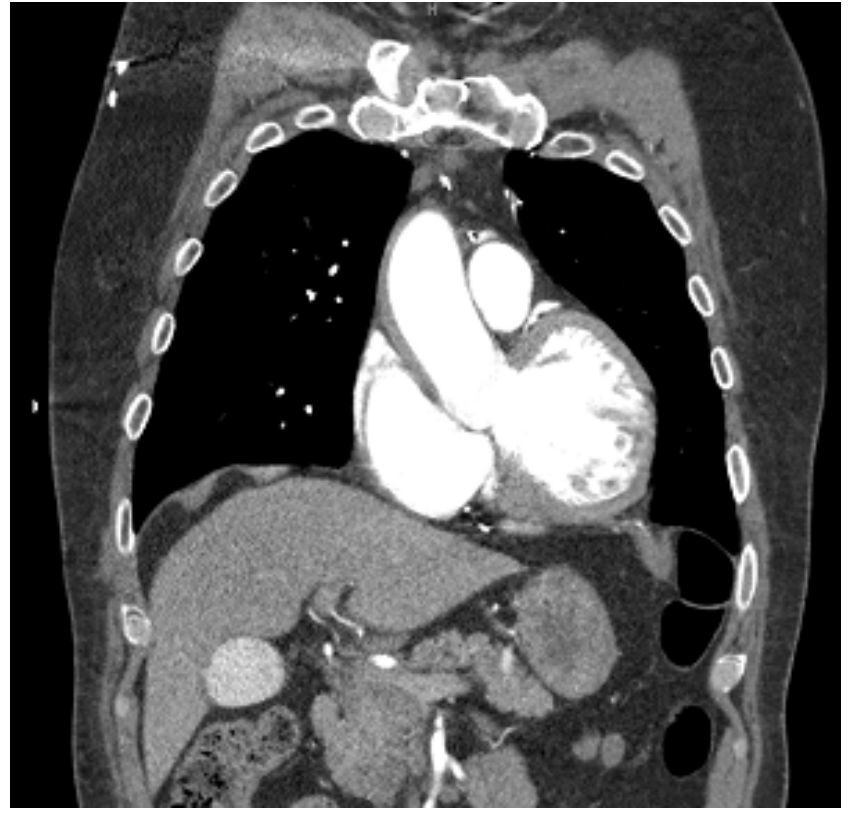

Figure 5: CT angiogram performed 12 hours after demonstrating near complete resolution of the false lumen of the ascending thoracic aortic dissection in comparison to Figure 2 and Figure 3, without evidence of aneurysm. No evidence of periaortic or mediastinal fluid.

discharged days later and remained stable and asymptomatic at his outpatient follow up appointments.

\section{Discussion}

latrogenic Stanford Type A aortic dissection is a rare but devastating and feared complication of coronary catheterization. In recent studies, the incidence of iatrogenic aortic dissection is as low as $0.02 \%$ [1]. Unfortunately, iatrogenic aortic dissection carries a mortality rate as high as $35 \%$, with mortality being even higher than that associated with non-iatrogenic aortic dissection (24\%) [2]. Known risk factors for coronary dissection with propagation into aortic root include older age, hypertension, and calcification of the aortic root [3].

Due to a scarcity of studies, there are no formal guidelines for the management of iatrogenic type $A$ aortic dissections. Most information we have on treatment modalities have come from case studies, which have given rise to two described management strategies. The less invasive treatment modality for this classification of dissection is coronary stent placement into the ostium of the RCA. The stent is placed to seal the entry site of the dissection and to attempt to preserve blood flow to the coronary vessel. The invasive surgical repair approach in type A aortic dissections bears a mortality rate of approximately $18 \%$ to $25 \%$ [3]. This approach is necessary in complicated aortic dissections involving the aortic root, presence of hemopericardium and tamponade physiology.

Intensive medical therapy is the commonly recommended initial medical treatment of thoracic aortic dissection to minimize shear forces by decreasing the $\mathrm{dp} /$ dt. This is achieved by reduction in heart rate and blood pressure; however, it is more dependent on the reduction of heart rate. As such, beta blockade for a goal hear rate less than $60 \mathrm{bpm}$, prior to initiating intravenous blood pressure reduction of nitroprusside or nitroglycerin is recommended.

In this case, the etiology of the dissection was likely the placement of a GuideLiner ${ }^{\circledR}$ catheter into the RCA and injection of this catheter into a CTO causing retrograde dissection. Because the patient had a prior median sternotomy and coronary artery bypass surgery, dense adhesions of the pericardium to the heart and ascending aorta were anticipated. Although the ascending aortic dissection appeared to start at the root of the aortic valve and ascended distally across to the innominate artery, the probability of retrograde progression of the dissection and rupture into the pericardial wall was deemed to be unlikely. Furthermore, a surgical intervention in a patient with prior CABG with a patent LIMA running close to the midline was considered an extremely high risk surgical procedure with high risk of mortality.

To our knowledge, this is the first reported case of a completely medically managed iatrogenic aortic dissection with radiographic evidence of complete resolution of the dissection. We also highlight the importance of CT angiography in the management of iatrogenic Type A dissections. Invasive cardiac angiography often underestimates the extent of dissection, as it did in this case. Alternatively, CT angiography is a less invasive and more reliable method of continuing to monitor dissections and has seen a significant increase in its utilization for diagnosis in recent years $[3,4]$. Lastly, our experience in this case demonstrates the potential for successful noninvasive medical treatment in a condition that was once considered to require a potentially life threatening surgical intervention.

\section{Sources of Funding}

This research did not receive any specific grant from funding agencies in the public, commercial, or not-forprofit sectors.

\section{References}

1. Pallav G, Buckley O, Rybicki F, Resnic F (2009) Resolution of iatrogenic aortic dissection illustrated by computed tomography. Circ Cardiovasc Interv 2: 261-263.

2. Januzzi JL, Sabatine MS, Eagle KA, Evangelista A, Bruckman D, et al. (2002) latrogenic aortic dissection. Am J Cardiol 89: 623-626.

3. Pape LA, Awais M, Woznicki EM, Suzuki T, Trimarchi S, et al. (2015) Long-Term trends in acute aortic dissection from the international registry of acute aortic dissection. Journal of the American College of Cardiology 66: 4.

4. Hiratzka LF, Bakris GL, Beckman JA, Bersin RM, Carr VF, et al. (2010) 2010 ACCF/AHA/AATS/ACR/ASA/SCA/SCAI/ SIR/STS/SVM Guidelines for the diagnosis and management of patients with thoracic aortic disease: Executive summary. J Am Coll Cardiol 55: 1509-1544. 